EUROPEAN ORGANIZATION FOR NUCLEAR RESEARCH

CERN-SL-2002-049 (AP)

\title{
Decoherence of a longitudinally kicked beam with chromaticity
}

\author{
G. Rumolo, F. Schmidt, R. Tomás.
}

\begin{abstract}
In this report a method to measure the chromaticity is presented. This method consists in applying a longitudinal kick and computing the Fourier transform of the transverse turn-by-turn BPM readings. An analytical expression for the Fourier transform is derived, which shows how the chromaticity can be inferred from the synchrotron sidebands. Finally, a multiparticle simulation is performed to validate the analytical expression.
\end{abstract}

Geneva, Switzerland

October 17, 2002 


\section{Introduction}

In presence of chromaticity the Fourier spectrum of the turn-by-turn transverse motion contains synchrotron sidebands around the fundamental tune. In principle, the measurement of these sidebands from the Fourier transform of beam position data could be used to determine the chromaticity. Nevertheless for a Gaussian beam centred in the longitudinal phase space this effect is quadratic in the chromaticity. Therefore the sign of the chromaticity cannot be inferred and the measurement of the smaller chromaticities requires much more accurate measurements. This problem is overcome if a longitudinal kick is applied to the beam, for instance, by shifting the phase of the RF cavities. In this case the amplitude of the sidebands contains a linear contribution in the kick and the chromaticity. The sign of the chromaticity is contained in the phase of the sidebands.

The analytical expressions relating the amplitude and phase of the sidebands with the longitudinal kick and the chromaticity are derived in this report. A Gaussian beam will be assumed for the derivations. A macroparticle simulation using the HEADTAIL [1] code is used to verify the analytical expressions.

\section{Derivation of the analytical expressions}

The initial density of a Gaussian bunch not centred in the longitudinal plane and matched to the bucket is given by

$$
\rho(\delta, z)=\frac{1}{2 \pi \sigma_{\delta} \sigma_{z}} e^{-\delta^{2} / 2 \sigma_{\delta}^{2}-\left(z-z_{\circ}\right)^{2} / 2 \sigma_{z}^{2}}
$$

where $\delta$ is the relative momentum deviation, $z$ is the longitudinal coordinate, $z_{\circ}$ is the initial longitudinal kick and $\sigma_{\delta}$ and $\sigma_{z}$ are the respective sigmas of the Gaussian distributions. The transverse tune as function of the turn number $N$ for a particle with initial coordinates $\delta$ and $z$ is expressed as

$$
\Delta Q_{x}=\frac{Q_{x}^{\prime}}{2 \pi Q_{s} N}\left[\delta \sin \left(2 \pi Q_{s} N\right)-z \frac{Q_{s}}{\eta R}\left(\cos \left(2 \pi Q_{s} N\right)-1\right)\right],
$$

where $Q_{x}^{\prime}$ is the horizontal chromaticity expressed as the derivative of the tune with respect to the relative momentum deviation, $R$ is the machine radius and $\eta$ is the slippage factor. The time evolution of one of the transverse coordinates of the centroid is given by averaging over the particle distribution:

$$
A(N)=\operatorname{Re}\left[\int_{-\infty}^{\infty} d z \int_{-\infty}^{\infty} d \delta \rho(\delta, z) e^{i\left(2 \pi\left(Q_{x}+\Delta Q_{x}\right) N\right)}\right] .
$$

By doing the double integral, $A(N)$ is expressed as:

$$
A(N)=\operatorname{Re}\left[e^{\left(\varsigma^{2}-i \varsigma k\right)\left(\cos \left(2 \pi Q_{s} N\right)-1\right)+i 2 \pi Q_{x} N}\right]
$$


where $\varsigma=Q_{x}^{\prime} \sigma_{\delta} / Q_{s}$ and $k$ is the longitudinal kick in units of $\sigma_{z}$. The Fourier spectrum of this expression is easily obtained by using the property

$$
e^{a \cos (\theta)}=\sum_{q=-\infty}^{\infty} I_{q}(a) e^{i q \theta}
$$

where $I_{q}(a)$ is the modified Bessel function of order $q$. Therefore,

$$
\begin{aligned}
A(N) & =\operatorname{Re}\left[e^{-\left(\varsigma^{2}-i \varsigma k\right)} \sum_{q=-\infty}^{\infty} I_{q}\left(\varsigma^{2}-i \varsigma k\right) e^{i 2 \pi\left(Q_{x}+q Q_{s}\right) N}\right]= \\
& =e^{-\varsigma^{2}} \sum_{q=-\infty}^{\infty}\left|I_{q}\left(\varsigma^{2}-i \varsigma k\right)\right| \cos \left(2 \pi\left(Q_{x}+q Q_{s}\right) N+\psi_{q}\right)
\end{aligned}
$$

where $\psi_{q}$ is the phase of the quantity

$$
I_{q}\left(\varsigma^{2}-i \varsigma k\right) e^{i \varsigma k}
$$

This expression provides the amplitude and phase of the synchrotron sideband of order $q$,

$$
\begin{aligned}
A M P(q) & =e^{-\varsigma^{2}}\left|I_{q}\left(\varsigma^{2}-i \varsigma k\right)\right|, \\
\operatorname{PHASE}(q) & =\psi_{q} .
\end{aligned}
$$

The amplitude contains a linear term in $\varsigma$ and $k$ but the sign of the chromaticity cannot be computed from the amplitude since

$$
\left|I_{q}\left(\varsigma^{2}-i \varsigma k\right)\right|=\left|I_{q}\left(\varsigma^{2}+i \varsigma k\right)\right|
$$

and $I_{q}(a)=I_{-q}(a)$. To obtain the sign of the chromaticity the phase of a sideband is needed. By inspecting the power expansion of the modified Bessel function in eq. (7) the following relation is inferred between the $\psi_{q}$ and $\psi_{0}$,

$$
\psi_{q}-\psi_{0}=-|q| \arctan \left(\frac{k}{\varsigma}\right) \text {. }
$$

Indeed, using this expression and knowing $k, \varsigma$ can be unambiguously determined from the phases of the sidebands.

\section{Analytical expressions versus simulation}

A macroparticle simulation has been done using an SPS model containing only chromaticity. Gaussian distributions of particles have been considered in the 


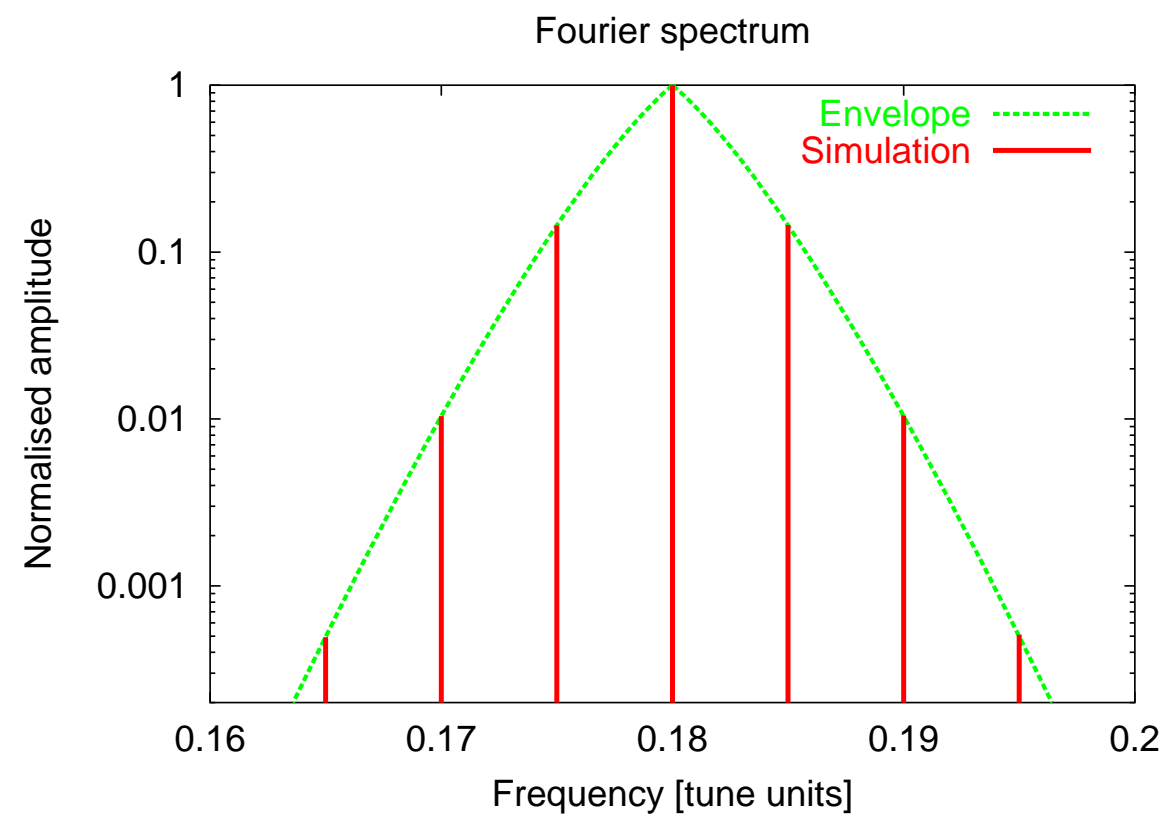

Figure 1: Normalised amplitude of the Fourier spectrum of the turn-by-turn simulated transverse position together with the predicted envelope function.

6-dimensional phase space. The longitudinal distribution is off-centred by the distance $z_{\circ}$. The parameters of the simulation are:

$$
\begin{aligned}
& Q_{s}=0.005 \quad, \quad \sigma_{\delta}=2.7210^{-3} \\
& Q_{x}=0.180 \quad, \quad \sigma_{z}=0.3 \mathrm{~m} \\
& Q_{x}^{\prime}=0.175, \quad z_{\circ}=3 \sigma_{z}
\end{aligned}
$$

From these quantities the two previously defined parameters needed for the evaluation of the analytical expressions are computed:

$$
\begin{aligned}
\varsigma & =Q_{x}^{\prime} \sigma_{\delta} / Q_{s}=0.095 \\
k & =z_{\circ} / \sigma_{z}=3 .
\end{aligned}
$$

The Fourier spectrum of the simulated transverse data is computed and compared to the analytical expressions. In figure 1 the normalised amplitude of the Fourier spectrum of the data is compared to the analytical expression of eq. (8). The agreement is excellent even for the weakest sidebands. In figure 2 the phase of the sidebands of the Fourier spectrum of the simulated data is compared to the analytical expression of eq. (7). Again, the agreement is excellent and the linear dependence of $\psi_{q}$ in $|q|$, predicted in eq. (11), is confirmed. 


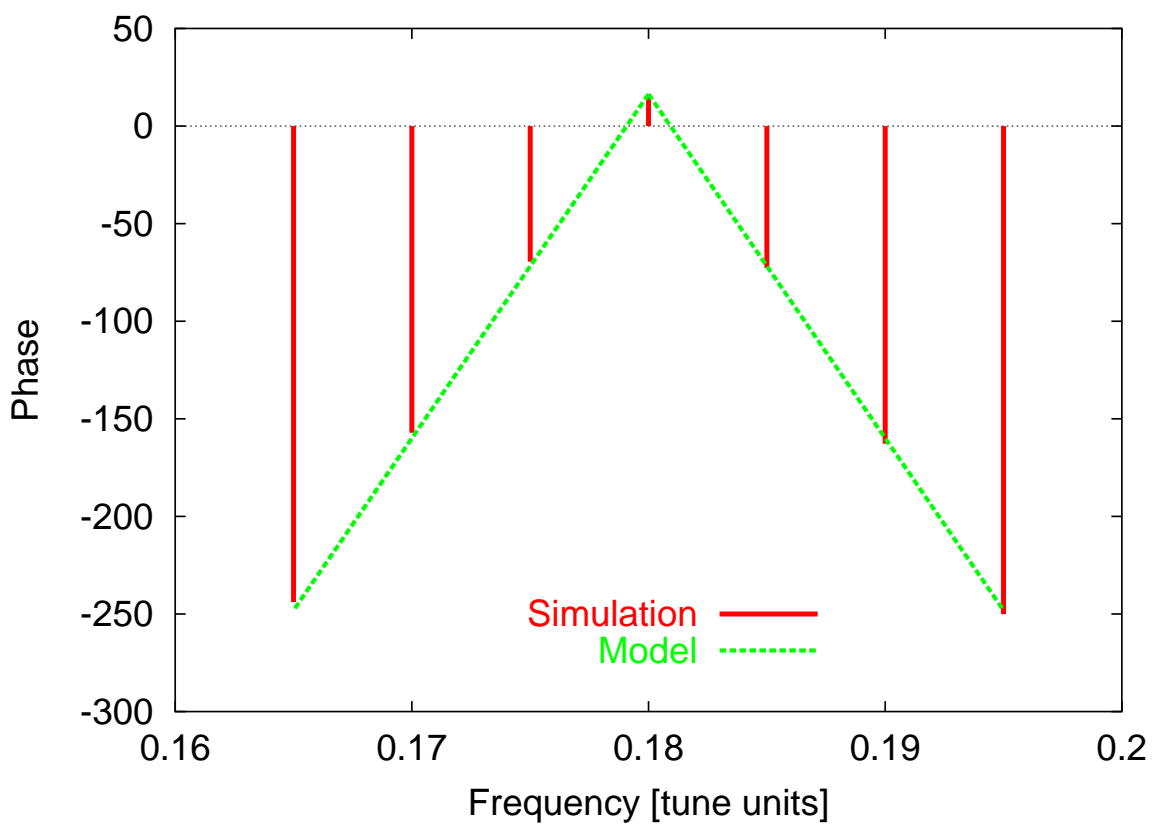

Figure 2: Phase of the sidebands of the Fourier spectrum of the turn-by-turn transverse position together with the predicted curve.

\section{Conclusion}

An analytical expression has been derived describing the Fourier spectrum of the turn-by-turn transverse position of the centroid of a Gaussian bunch longitudinally kicked. Using this expression the chromaticity of an operating accelerator could be measured by shifting the RF phase (equivalent to an energy kick) and Fourier analysing the transverse beam position data. The analytical derivation has been compared to a macroparticle simulation obtaining an excellent agreement.

\section{Acknowledgements}

The authors thank Dr. Stephane Fartoukh for help in carrying out the mathematical derivations. We are also thankful to Dr. Oliver Brüning, Dr. Francesco Ruggiero and Dr. Frank Zimmermann for reading the manuscript and making valuable comments.

\section{References}

[1] G. Rumolo, F. Zimmermann, "Electron cloud simulations: beam instabilities and wake fields" ECloud'02 proceedings CERN-2002-001. Programe 
documentation at http://wwwslap.cern.ch/collective/electron-cloud/electroncloud.html 